\title{
Bacterial Meningitis Complicated by Venous Sinus Thrombosis: a Case of a Pregnant Woman
}

\author{
Petek Konya ${ }^{1}$ (D), Neşe Demirtürk ${ }^{1}$ (ID \\ 1 Department of Infectious Diseases and Clinical Microbiology, Afyonkarahisar University of Health Sciences, \\ Afyonkarahisar, Turkey
}

\begin{abstract}
Bacterial meningitis is a severe infectious disease resulting in high mortality and morbidity throughout the world. The causative pathogens of bacterial meningitis depend on the patient's age and predisposing factors. A 23-year-old pregnant woman at 36 weeks of gestation was admitted to the emergency unit with confusion and fever. She was lethargic, uncooperative, disoriented, and had been diagnosed with acute otitis media by a family physician. She had been prescribed oral amoxicillin-clavulanate and sent home. When she applied to the emergency unit, purulent drainage was obtained from her right ear. Magnetic Resonance Venogram showed filling defects affecting the jugular vein and right mastoid posterior sigmoid sinus. Gram-positive cocci and chains were seen in the direct examination of CSF.

Results: This report shows that otitis media should be treated carefully and followed to prevent life-threatening complications during pregnancy. If optimal antibiotic therapy is not given in time for bacterial meningitis that develops during pregnancy, the disease can be fatal for the mother and child.
\end{abstract}

Keywords: Bacterial, meningitis, otitis media, Gram-positive, Pregnant.

\section{INTRODUCTION}

$B$ acterial meningitis is a severe infectious disease resulting in high mortality and morbidity throughout the world. The causative pathogens of bacterial meningitis depend on the patient's age and predisposing factors such as after splenectomy or with a hyposplenic state, chronic kidney or liver disease, HIV infection, alcoholism, hypogammaglobulinaemia, diabetes mellitus and patients using immunosuppressive drugs (2).

The prevalence of bacterial meningitis in pregnant women is very low. Only 48 case reports were published in this topic around the world until April 2012. Also, the most common causative agents were Streptococcus pneumoniae and Listeria monocytogenes (3).

\section{Corresponding Author: Petek Konya \\ E-mail: \\ nesed60@hotmail.com}

Received: January 20, 2020

Accepted: May 25, 2020

Published: November 20, 2020

\section{Suggested citation:} Konya P, Demirtürk N. Bacterial Meningitis Complicated by Venous Sinus Thrombosis: a Case of a Pregnant Woman. Infect Dis Clin Microbiol 2020; 3 : 168-170.

DOI: 10.36519/idem.2020.0001 


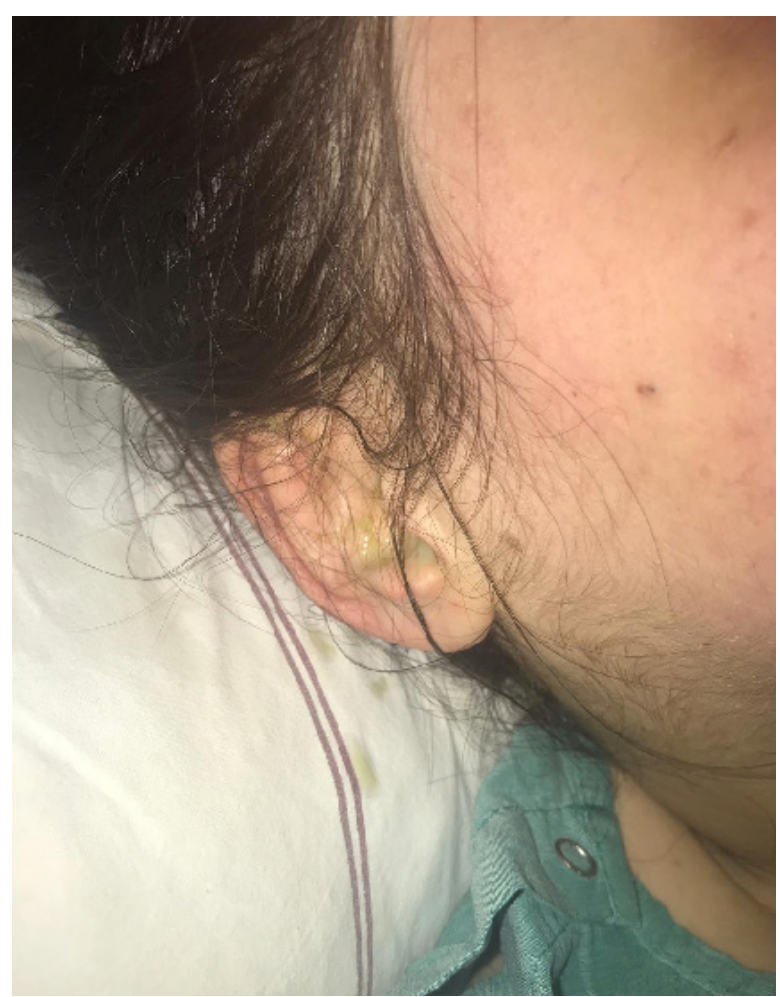

Figure 1. Purulent drainage from right ear.

In order to protect the fetus from an immunological attack by the mother's immune system, immunosuppressive cytokines are produced by the placenta during pregnancy. However, it does not lead to increased susceptibility to most infectious diseases, including pneumococcal disease, and usually, infections in pregnant women are not more severe than in non-pregnant women (4). Cerebral venous thrombosis (CVT) is described as thrombosis of the superficial or deep venous system (5). Vascular complications are common in bacterial meningitis, but venous sinus thrombosis is rare (6). The incidence of CVT increases with sex-specific risk factors such as pregnancy and puerperium (7). In this study, we reported bacterial meningitis complicated by venous sinus thrombosis in a pregnant woman.

\section{CASE REPORT}

A 23-year-old pregnant woman at 36 weeks of gestation was admitted to the emergency unit with confusion and fever. She was lethargic, uncooperative, and disoriented. Her family report- ed that she had applied to a family physician a week ago with the complaint of right otalgia. The diagnosis of acute otitis media had been made, and treatment with oral amoxicillin-clavulanate had been started in the outpatient clinic. On physical examination in the emergency unit, she was febrile $\left(38.7^{\circ} \mathrm{C}\right)$ and had tachycardia and tachypnea, accompanied by neck stiffness. Purulent drainage was obtained from her right ear (Figure 1). She was becoming progressively unconscious. However, there was no uterine contraction and cervical dilation. Laboratory analysis showed moderate anemia (hemoglobin $11.0 \mathrm{~g} / \mathrm{dl}$ ). Complete blood count revealed a leukocytosis of $22 \times 10^{\wedge} 3 /$ uL with $90 \%$ neutrophil, and platelet count was 396000/Ul. The C-reactive protein (CRP) level was $6.8 \mathrm{mg} / \mathrm{dL}$, and the erythrocyte sedimentation rate was $95 \mathrm{~mm} /$ hour. Liver and kidney function tests were normal.

Magnetic Resonance Imaging (MRI) of the brain and Magnetic Resonance Venogram (MRV) showed filling defects affecting the jugular vein and right mastoid posterior sigmoid sinus. Low-molecular-weight heparin was started for sigmoid sinus venous thrombosis.

The fetus was in vertex presentation with a size consistent for gestational age and normal amniotic fluid. Fetal heart tones were noted to be 175bpm. An emergency caesarean section was applied. 2100 gr baby girl was born with APGAR scores 8-9. Lumbar puncture was performed in the operating room immediately after caesarean section. The cerebrospinal fluid (CSF) was turbid with 10,000 leucocytes $/ \mathrm{mm}^{3}$ (95\% polymorphs). The CSF sugar was below normal limits 27 (50-80) mg/100 ml (meanwhile, blood glucose was 80), and proteins were $176 \mathrm{mg} / 100 \mathrm{ml}$. Gram-positive cocci and chains were seen in the direct examination of CSF. Blood cultures and CSF had been performed before the treatment was started.

She was transferred to the intensive care unit after the operation. She was initially treated with ceftriaxone (4g/day) and vancomycin (2g/day) combination therapy empirically. On the second day of treatment, the patient's consciousness improved, and fever decreased. The first blood and CSF cultures obtained prior to antibiotic therapy 
were also positive for S pneumonia. According to the antibiogram, the causative agent was sensitive to vancomycin and ceftriaxone. Antibiotic treatment was completed to 10 days, and the patient was discharged with low molecular weight heparin. She was followed up in the neurology department regularly after the discharge from the hospital, and her recovery was good.

\section{DISCUSSION}

Venous sinus thrombosis is a common and severe complication in adults with bacterial meningitis (6). Underlying hormonal factors such as pregnancy and infections increase the risk of thrombosis (8). Venous sinus thrombosis secondary to infection may not be indistinguishable from stroke and therefore requires clinical suspicion to make an accurate diagnosis. Preventing venous sinus thrombosis will be important in reducing the high morbidity and mortality rate in adults with bacterial meningitis.
Bacterial meningitis during pregnancy is rare. In our literature review, although we found some case reports and a small case series of women diagnosed with pneumococcal meningitis during pregnancy or the postpartum period, none of them was concomitant with SVT. Lucas described 26 cases of pneumococcal meningitis in Nigerian during pregnancy or the immediate postpartum period (9). Adriani et al. identified six cases of community-acquired pneumococcal meningitis during pregnancy in the Netherlands in 6 years (4). In these studies, otitis and sinusitis were the most common predisposing conditions.

This study shows that otitis media should be treated carefully and followed to prevent life-threatening complications during pregnancy(10). If optimal antibiotic therapy is not given in time for bacterial meningitis that develops during pregnancy, the disease can be fatal for the mother and child.
Informed Consent: Written consent was obtained from the patient.

Peer-review: Externally peer-reviewed

Author Contributions: Concept - P.K.; Design - ALL; Supervision - ALL; Materials - ALL; Data Collection and/or Processing - ALL; Analysis and/or Interpretation - N.D.; Literature Review - N.D.;
Writer - ALL; Critical Reviews - ALL.

Conflict of Interest: The authors have no conflict of interest to declare.

Financial Disclosure: The authors declared that this study has received no financial support.

\section{REFERENCES}

1 van de Beek D, Cabellos C, Dzupova O, Esposito S, Klein M, Kloek AT, et al. ESCMID guideline: Diagnosis and treatment of acute bacterial meningitis. Clin Microbiol Infect 2016; 22: S37-62.

2 Brouwer MC, Tunkel AR, van d Beek D. Epidemiology, diagnosis, and antimicrobial treatment of acute bacterial meningitis. Clin Microbiol Rev 2010; 23: 467-92.

3 Scharek P, Jekkel C, Budai J, Szilasi Z, Helferich F, Arva I, et al. Pneumococcal meningitis in a pregnant woman. Ideggyogy Sz 2014; 67: 56-8.

4 Adriani KS, Brouwer MC, van der Ende A, van de Beek D. Bacterial meningitis in pregnancy: report of six cases and review of the literature. Clin Microbiol Infect 2012; 18: 345-351.

5 Selvitop O, Poretti A, Huisman TAGM, Wagner MW. Cerebral sinovenous thrombosis in a child with Crohn's disease, otitis media, and meningitis. Neuroradiol J 2015; 28: 274-7.

6 Xie W, Liu Q, Feng J, Fang S. A case of bacterial meningitis complicated by venous sinus thrombosis. Neurol Sci 2015; 36: $331-2$.

7 Kashkoush AI, Ma H, Agarwal N, Panczykowski D, Tonetti D, Weiner GM, et al. Cerebral venous sinus thrombosis in pregnancy and puerperium: A pooled, systematic review. J Clin Neurosci 2017; 39: 9-15.

8 Kaya D. The Diagnosis and treatment of cerebral venous thrombosis. Turk J Neurol 2017; 23: 94-104.

9 Lucas S. Acute bacterial meningitis during and after pregnancy. BJOG 2012; 119: 1555-7.

10 Landrum LM, Hawkins A, Goodman JR. Pneumococcal meningitis during pregnancy: A case report and review of literature. Infect Dis Obstet Gynecol 2007; 2007: 39-41. 